\title{
COMMUNICATION TRAFFIC CHARACTERISTICS OF SMART GRID FASTADR AGGREGATION FOR DISTRIBUTED OFFICE BUILDINGS' LOADS
}

\author{
Chuzo Ninagawa ${ }^{1}$ and Keita Suzuki ${ }^{2}$ \\ ${ }^{1}$ Dept. of Electrical and Electronic Eng., Gifu University, Gifu, Japan \\ ${ }^{2}$ Interlink, Inc., Nagoya, Japan
}

\begin{abstract}
This paper gives emulation studies on the communication traffic characteristics of the future Smart Grid's Fast Automated Demand Response (FastADR) Aggregation for widely-distributed office buildings' facility loads. A real-time realistic emulation experiment system was developed consisting of 80 devices in which actual FastADR aggregation communication software was installed. The Internet traffic conditions were emulated by burst IP packet losses and round trip time delays. Our experimental studies showed more severe variation of aggregation data transmission time than expected before. In an example case of power curtailment, i.e., negawatt of $5 \mathrm{MW}$ from 50 buildings, more than 60 seconds will be needed to complete a sequential aggregation web services with each building's gateway.
\end{abstract}

\section{KEYWORDS}

Communication Traffic, WAN, Web Services, Smart Grid, Automated Demand Response, Aggregation

\section{INTRODUCTION}

Smart Grid technologies for the future power systems are gaining attentions recently. Among these technologies, Automated Demand Response (ADR) technology is important.Traditionally, Demand Responses have been treated as a slow, hourly or daily management method. In the last few years, however, some possibilities of the FastADR have been studied for ancillary services of several minutesorder timescales.

Office buildings' air-conditioning facilities are suitable target loads for the FastADR because of the large volume of power demand and less affection to the residents' activities. This paper deals with a large number of package type building air-conditioners in the buildings located over the wide areas as the target loads of the FastADR aggregation.

As shown in Figure 1, one of the components of the FastADR system is the Aggregator service provider which divides the ADR requirement to each small customer's loads and collects the resulting power curtailments. In this examplesystem, the Aggregator communicates with each Building Energy Management System (BEMS) Gateway for the FastADR aggregation.

So far many research works on the FastADR have been carried out[1]-[3]. Some research works investigated delay characteristics of the building facility loads to react the FastADR[4]-[6]. A few studies reported the aggregation result measurementerror due to coarse sampling-time. Those are all studies on aggregation characteristics of the target loads.

Few research studies have dealt with the WAN communication delay problem in the FastADR Aggregation in the case of aggregation from a large number of customer loads spread over wide areas[7][8]. Even in the case of the FastADR aggregation from tens of thousands of retail shops' facilities over the almost half of the country, the WAN communication delays were neglected.

DOI: $10.5121 /$ ijcacs.2016.1201 
However, a few recent research works pointed out the importance of the WAN communication

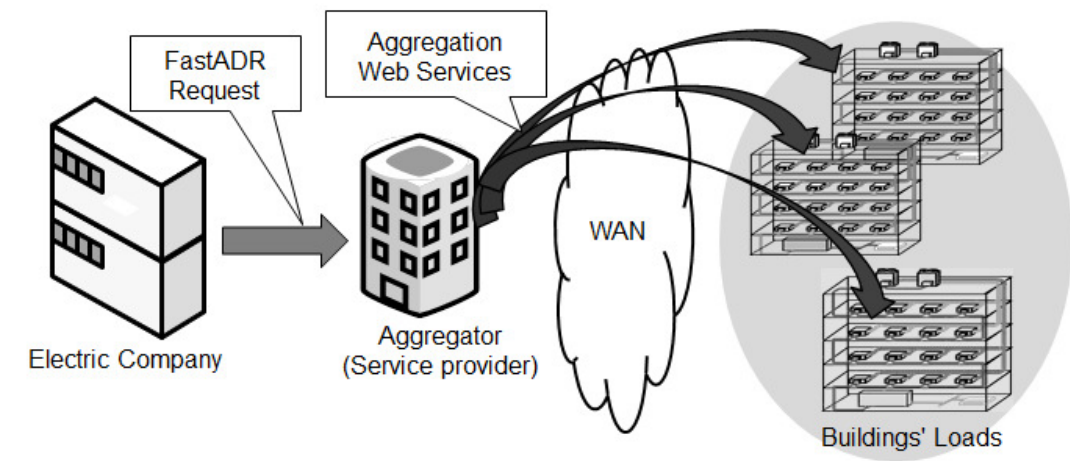

Figure 1. The conceptual diagram of the Fast Automated Demand Response Aggregation System for a widely-distributed buildings' facility loads.

delay for the FastADR[9][10]. Especially, in cases of feedback control are applied to the FastADR Aggregation for a large number of customersconnected with the WAN communications severe problems may occur. For example, the control characteristics of the FastADR power curtailment possibly shows severe unstablebehaviour due to unexpected large dead time in the digital control feedback loop[11][12].

In this research work, communication traffic characteristics of the FastADR Aggregation for widely-distributed office buildings' facility loads. A real-time realistic emulation experiment system was developed, and the Internet traffic conditions were emulated by burst IP packet losses and round trip time delays. Our experimental studies showed variation of aggregation data transmission time than expected before. In an example case of power curtailment, i.e., negawatt of $5 \mathrm{MW}$ from 50 buildings, more than 60 seconds will be needed to complete a sequential aggregation web services with each building's gateway.

\section{FASTADR AGGREGATION}

\subsection{Aggregation Communication System Design}

Figure 2 shows a communication system architecture of the FastADR Aggregation system. The FastADR centre of an electric company is equipped with a Demand Response Automation Server (DRAS). The Aggregator's subsystem includes the Controller, Aggregation Server, and Report Storage.

The Controller plays a role of a client for the DRAS server to receive a FastADR Request using a specializedcommunication protocol such as OpenADR 2.0 standard[13]. Once the Controller receives the FastADR Request, it divides the requested amount into each building's power curtailment command and writes them to the Aggregation Server.

The Aggregation Server distributes these power curtailment commands using Aggregation Web Services using communication protocol for wide-area monitor and control of facility loads. In this research work, we employed the IEEE1888 communication standard[14]-[16]for the Aggregation Web Services. Since the FastADR customer building may be located wide area, the communication channel of the Aggregation Web Services will be the Internet based wide area 
network (WAN). Web service based communication protocols such as the IEEE1888 are suitable for monitor and control through firewalls and other restrictions for widely distributed customer communication.

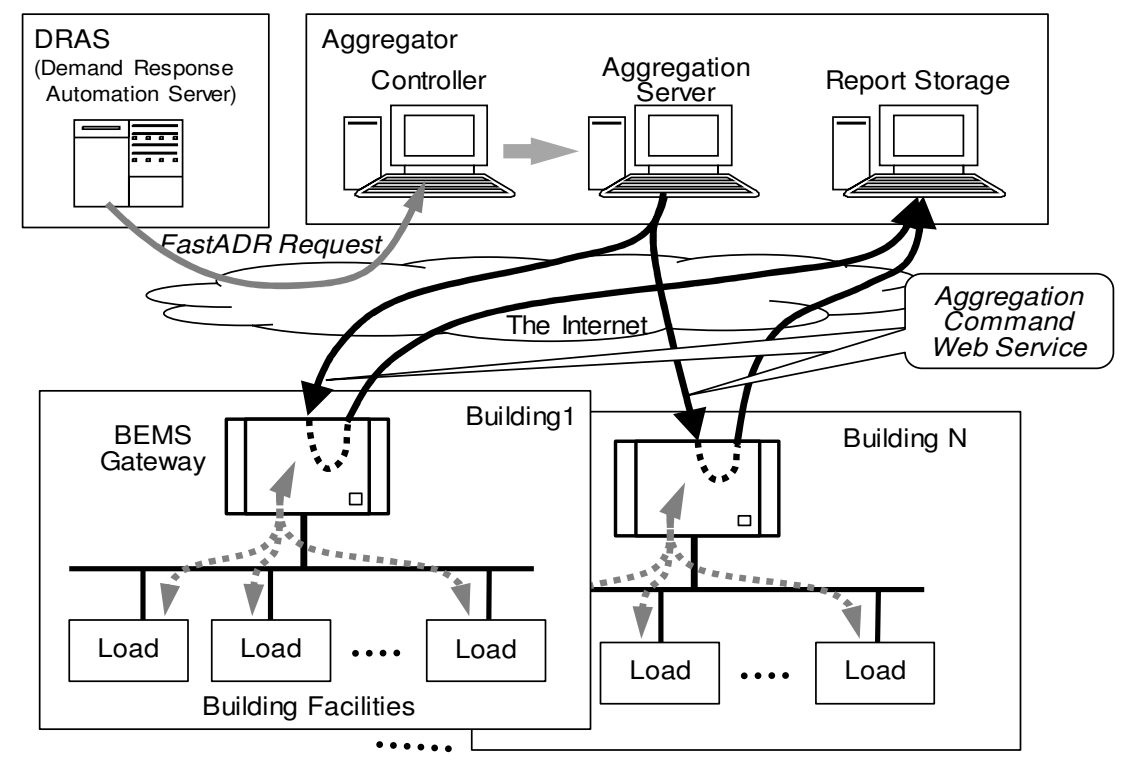

Figure 2. Communication system diagram of the FastADR aggregation for widely-distributed building facility loads

Each customer building has a Building Energy Management System (BEMS) Gateway, which not only communicates with the Aggregation Server but also controls the power consumption of the building's facility loads, such as air-conditioners, lightings, and so on. The BEMS Gateway receives the Aggregation Web Services and converts them to fieldbus command for power curtailment of each load. Also, the BEMS Gateway sums up the power curtailments to send them back to the Report Storage of the Aggregator. We call this pair of command and report data transmissions as an AggregationWeb Service.

\subsection{Communication Sequence Chart}

Figure 3 shows the communication sequence chart of our example FastADR Aggregation system. This communication sequence design is not just example, but we believe that this selection of chain of the communication protocol standards are most suitable for the FastADR Aggregation WAN communications.

The communication between the DRAS and the Controller is linked by the OpenADR $2.0 \mathrm{~b}$ standard. This part of the communication often uses the proprietary fast and reliable network and consequently the communication delay problems will not occur.

The problematic part of the FastADR Aggregation communication system is between the Aggregation Server and each BEMS Gateway. This part is should be less expensive and less effort to setup. In the case of widely-distributed office building's facility loads throughout the inter-cities areas, the most economical communication channel will be the Internet Virtual Private Networks (VPNs). If the system uses the Internet, practically only way is HTTP based Web 
Services communication protocols because these protocols assures the ability to pass through the firewall of target buildings and interoperability with each BEMS Gateway.

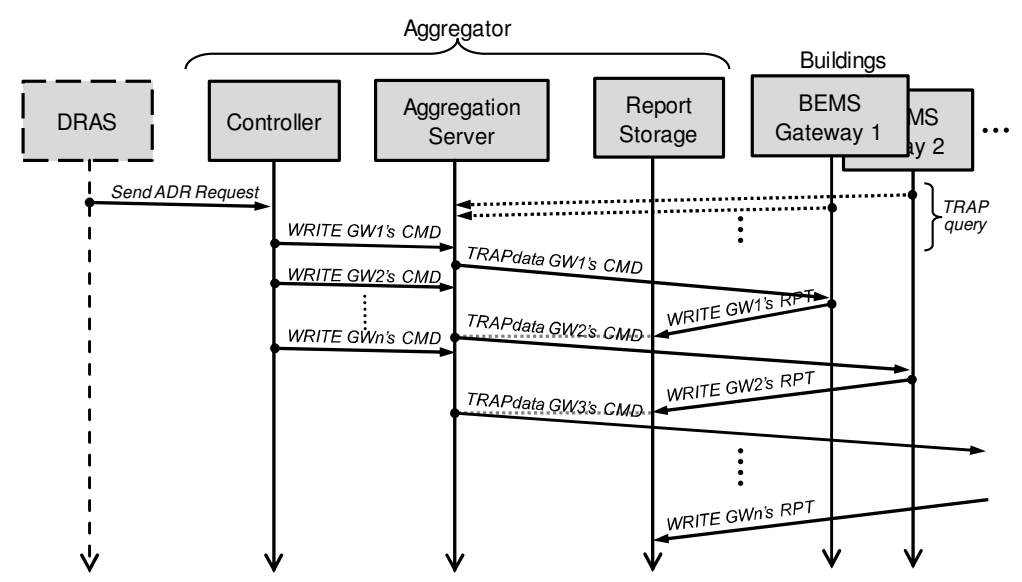

Figure 3. Communication sequence chart of the FastADR Aggregation system for a large number of BEMS Gateways

Therefore, for the communication protocol between the Aggregation Server and each BEMS Gateway a web service based IEEE1888 protocol that is specialized for facility monitor and control was chosen. The Aggregation Server pushes the Aggregation command to each BEMS Gateway using the IEEE1888's TRAP WRITE method. This method makes it possible so called "server-push" notification of the event occurrence on the server side.

\section{Aggregation Communication Experiment}

\subsection{Realistic Communication Experiment System}

In order to aggregate the FastADR power curtailments up to a meaningful amount, such as an order of 10 [MW], the aggregator will have to communicate with hundreds of buildings' BEMS Gateways. The total number of loads might be more than thousands widely spread all over the city. The FastADR aggregation experiments using huge number of actual buildings' loads will not be possible. Therefore, we constructed a realistic FastADR Aggregation communication experiment system in our laboratory.

Although the experiment system can simulated only communication part of the total latency of FastADR, it is significant part in the case of aggregation of wide area distributed building facility loads.

Figure 4 shows the hardware system diagram of our realistic FastADR communication experiment system. The Aggregation Server PC is connected with a cluster of WAN emulator "Dummynet"[17] devices using the lab LAN controller. Each Dummynet device is connected with a BEMS Gateway. Then, each BEMS Gateway is connected with a Building Facility Load Emulator. 


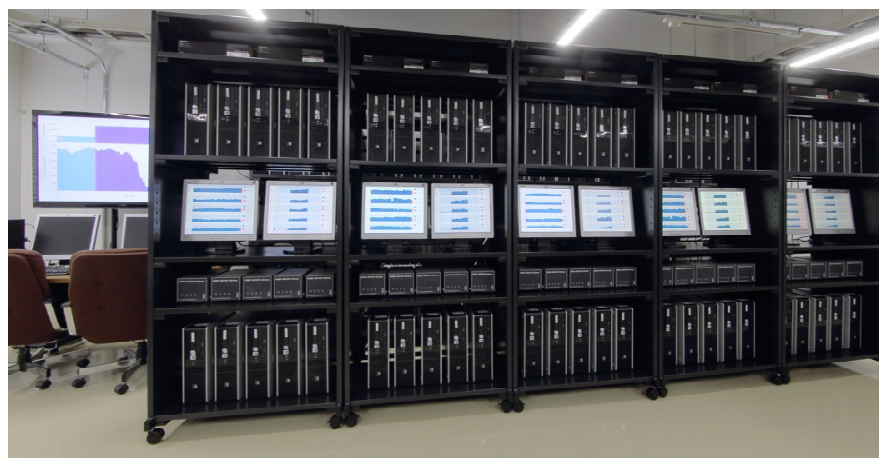

(a) Appearance

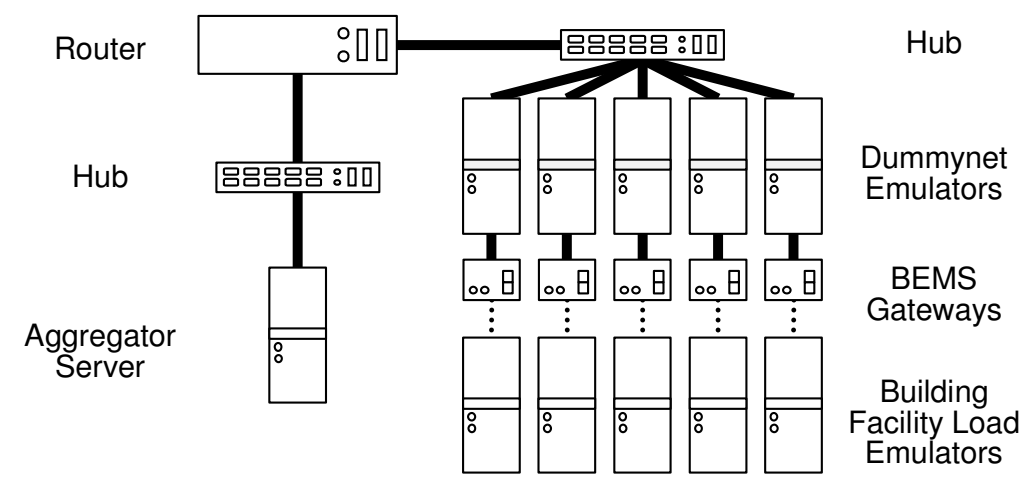

(b) Hardware system diagram

Figure 4. Realistic communication experiment system.

The Building Facility Load Emulator play a role of facility power consumption behaviours, such as change of power consumption according to the FastADR Aggregation power curtailment command as if the actual loads are operating.

\subsection{Packet Delay and Loss Emulator}

The Internet data transmission condition varies significantly time to time, place to place. It is almost impossible to construct a model of data transmission delay from the measurements using the actual Internet. Instead, we chose a test-bench approach in the laboratory instead of fieldtesting using the real Internet.

Figure 5 shows the mechanism of "Dummynet" IP packet loss and delay emulator. The emulator has two network interface cards (NICs). Between the two NICs, we set a pair ofqueues for the both ways emulating the link bandwidth, transmission delay, and packet loss rate. The probability of packet loss is set to be uniformly random, that is, bursty using the Gilbert Elliot loss model[18][19].

The state transition diagram in Figure 5 shows the mechanism of generating burst occurrences of packet loss of Gilbert Elliot model. In the case of the Normal State, incoming IP packets are dropped at random with a negligible small probability, in our case, $p_{N}=0$. However, once the state transition occurs to the Severe State, incoming IP packets are dropped with a large 
probability, in our case, $p_{S}=0.5$. By tuning the state transition probability, the total average loss rate $P L$ was set as $P L=2[\%]$ in our work.

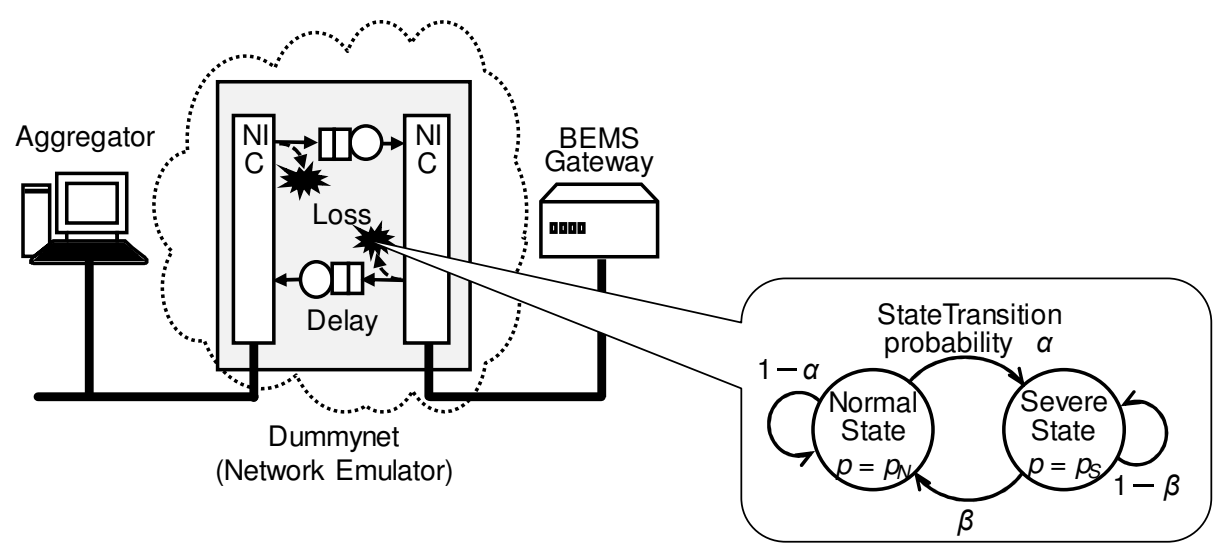

Figure 5. Mechanism of "Dummynet" that emulated stochastic generation of IP packet transmission RTT delays and the Gilbert Elliot model burst packet losses.

\section{EXPERIMENT RESULTS}

\subsection{Data Transmission Time Variation}

We carried out the data transmission experiments for the case of fast and slow WAN, where the average IP packet round trip time was $R T T=10$ and 100 [ms], respectively. Figure 6 shows experiment results of the data transmission time $T_{D T}$ of a single pair of Aggregation Command and Aggregation Report Web Servicesbetween the Aggregation Server and each BEMS Gateway. Since the Aggregation Web Services use the TCP/IP protocol over the WAN, the $T_{D T}$ varies stochastically at every data transmission. More than 1000 times of the pair of Aggregation Web Services were carried out to investigate the variation of $T_{D T}$.

Figure 6(a) shows the experiment results in the case of the most desirablecondition of fast round trip time, $R T T=10[\mathrm{~ms}]$ and packet loss free, i.e., $P L=0 \%$. The round trip time $R T T=10$ [s] was chosen as a typical Internet $R T T$ value between cities measured in our previous research work. Almost all $T_{D T}$ s were less than 1 second.

Figure 6(b) is the case of fast but lossy WAN of $R T T=10$ [ms] and $P L=2[\%]$. The value of PL $=2[\%]$ is an example of the average IP packet loss rate measured in our previous research work. In this case, the minimum $T_{D T}$ was as same as the case of $\mathrm{PL}=0$ [\%], but the variation reached approximately $10[\mathrm{~s}]$.

Then we carried out experiments for the case of slow WAN, where the average IP packet round trip time was $R T T=100$ [ms]. This condition is for the case of complicated and long haul WAN. Figure 6(c) shows the experiment results in the case of a slow round trip time, $R T T=100$ [ms], but packet loss free, i.e., $P L=0[\%]$. The variation of $T_{D T}$ s was as small as the fast and packet loss less case. 


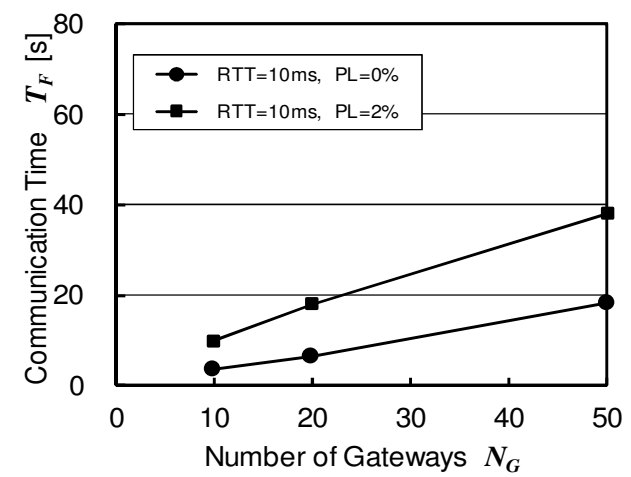

(a) Fast WAN : RTT $=10[\mathrm{~ms}]$

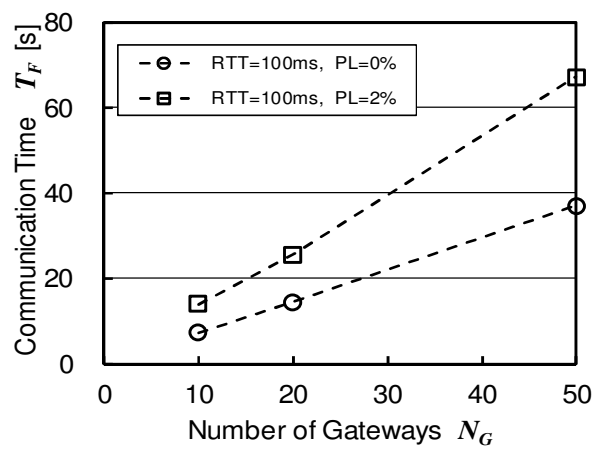

(b) Slow WAN : RTT $=100[\mathrm{~ms}]$

Figure 7. Scalability of the FastADR Aggregation communication as a function of the number of BEMS Gateways for a single Aggregation Server.

(c) Case 3: RTT = $100[\mathrm{~ms}], \mathrm{PL}=2[\%]$
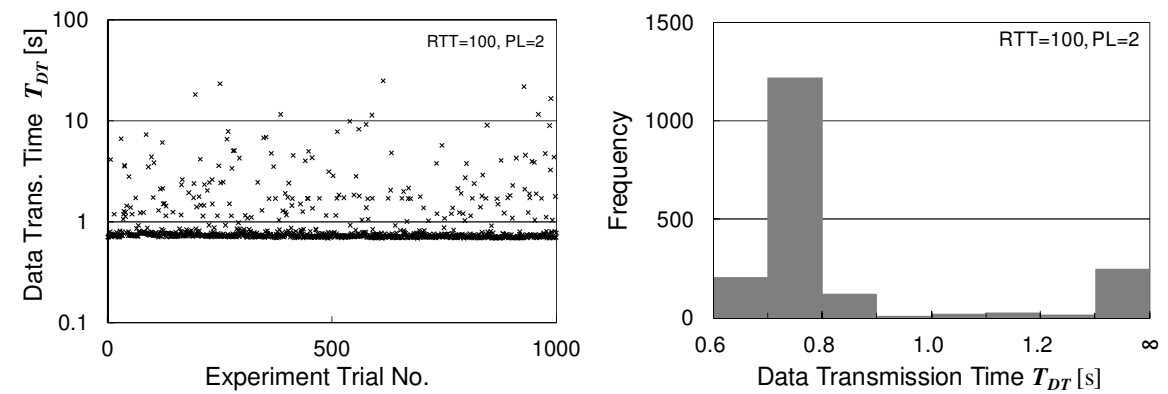

(d) Case 4: RTT = $100[\mathrm{~ms}], \mathrm{PL}=2[\%]$

Figure 6. Experiment results of the variation of Aggregation data transmission time from the Aggregation Server to the BEMS Gateway in the case of a short RTT.

As shown in Figure 6(d), however, in the case of a slow and packet lossy WAN, that is, $R T T=$ 
$100[\mathrm{~ms}]$ and $P L=2[\%]$, the variation of $T_{D T} \mathrm{~s}$ became significantly large. The minimum $T_{F}$ was

\subsection{Scalability of Aggregation Communication}

Figure 7 shows the increase of total communication time $T_{F}$ of the sequential FastADR aggregation Web Services for all the BEMS Gateways under its management along with the number of BEMS Gateways $N_{G}$ increases. The total communication time $T_{F}$ was defined as the average of 100 experiment trials of the Aggregation Web Services communication. A single round of the Aggregation Web Services communication was a sequence from the first pair of Aggregation Web Service data transmission between the Aggregator and the first BEMS Gateway, then the second, and so on to the last Gateway.

Figure 7(a) is for desirable WAN condition cases of a short IP packet round trip time $R T T=10$ [ms] and the average packet loss rate $P L=0$ or 2 [\%] of the Gilbert Elliot loss model.

Figure 7(b) shows an example case of power curtailment, i.e., negawatt of 5 MW from 50 buildings, more than 60 seconds will be needed to complete a sequential Aggregation Web Services with each building's gateway.

\section{DisCUSSION}

In this research, we used so called "server-push" communication technology by adopting the TRAP service method of the IEEE1888 Web Services protocol standard. After that the BEMS Gateway only needs to wait for the event-driven Aggregation Web Service request notification from the Aggregation Server. This makes it possible for each Aggregation Web Service data transmission time approximately 1 seconds for a desirable WAN condition of $R T T=10[\mathrm{~ms}]$ and $P L=0[\%]$.

However, if such a "server-push" technology is not applicable, each BEMS Gateway has to fetch the Aggregation command from the Aggregation Server periodically by using an ordinary"clientpull" technology. As a result, the Aggregation Web Service data transmission time will be significantly large and stochastic. The data transmission time varies at random from the minimum time that is equal as the server-push case to the maximum time of the fetch interval.

From the communication technology's point of view, each Aggregation Web Service data transmission could be done parallel instead of the sequential way shown in the previous section. However, in the case of FastADR Aggregation, the Aggregation Server is required to accumulate the FastADR power curtailment amount in each Web Service response from each BEMS Gateway one by one. If the Aggregator Server initiate the Aggregation Web Service request all in once, the sum of power curtailment from the Aggregation Web Service responses might undershoot or overshoot the target value. Therefore, the Aggregation Server is needed to send the Aggregation Web Service one gateway by one gateway sequentially. Parallelization could be done by multiple Aggregators at the OpenADR communication part between the DRAS server and the Controller of each Aggregator.

\section{CONCLUSIONS}

This paper has studied on the communication traffic characteristics of the future Smart Grid's Fast Automated Demand Response (FastADR) Aggregation for widely-distributed office buildings' facility loads. A real-time realistic emulation experiment system was developed consisting of 80 devices in which actual FastADR aggregation communication software was installed. The Internet traffic conditions were emulated by burst IP packet losses and round trip time delays.

Our experimental studies showed more severe variation of aggregation data transmission time than expected. In an example case of power curtailment, i.e., negawatt of $5 \mathrm{MW}$ from 50 
buildings, more than 60 seconds will be needed to complete a sequential aggregation web services with each building's gateway.

\section{REFERENCES}

[1] O. Ma, N. Alkadi, P. Cappers, P. Denholm, J. Dudley, S. Goli, M. Hummon, S. Kilcote, J. MacDonald, N. Matson, D. Olsen, C. Rose, M. Sohn, M. Starke, B. Kirby, \&M .O'malley, "Demand Response for Ancillary Services", IEEE Trans. on Smart Grid, Vol. 4, No. 4, pp1988-1995, 2013.

[2] H. Hao, Y. Lin, A. S. Kowli, P. Barooah, and S. Meyn, "Ancillary Service to the Grid Through Control of Fans in Commercial Building HVAC Systems,” IEEE Trans. Smart Grid, vol. 5, no. 4, pp. 2066-2074, 2014.

[3] Y. Lin, P. Barroah, S. Meyen, and T. Middlekoop, "Experimental Evaluation of Frequency Regulation From Commercial Building HVAC Systems", IEEE Trans. on Smart Grid, Vol. 6, No. 2, pp.776-783, 2015.

[4] C. Ninagawa, S. Kondo, S. Isozumi, and H. Yoshida : "Fine-time-granularity fast demand control of building HVAC facilities for future smart grid," in Proc. IEEE Int. Conf. on Innovative Smart Grid Technologies, ISG T Europe 2012, pp. 1-6, 2012.

[5] K. Suzuki and C. Ninagawa, "Communication Characteristics of Smart Grid ADR Aggregation for Building Facility Power Consumption," in Proc. Institute of Electrical Installation Engineers Japan International Workshop 2013, pp.545-548, 2013.

[6] K. Suzuki, C. Ninagawa, H. Yoshida, S. Kondo, J. Morikawa, T. Kanbe and Takao Aoki : "Smart Grid ADR Aggregation Delay Model on Large-Scale Distributed Building HVAC Facilities", in Proc. IEEE Innovative Smart Grid Technologies, ISGT Europe 2013, 2013.

[7] C. Ninagawa, H. Yoshida, S. Kondo, and H. Otake, "Data Transmission of IEEE1888 Communication for Wide-area Real-time Smart Grid Applications," International Renewal and Sustainable Energy Conference IRSEC'13, pp.1-6, 2013.

[8] C. Ninagawa, T. Iwahara, and K. Suzuki, "Enhancement of OpenADR Communication for Flexible Fast ADR Aggregation Using TRAP Mechanism of IEEE1888 Protocol", in Proc. IEEE Int. Conf. on Industrial Technologies, ICIT2015, Seville, Spain, 2015.

[9] U. Herberg, D. Mashima, J.G. Jetcheva, S. Mirzazad-Barijough, "OpenADR 2.0 deployment architectures: Options and implications," IEEE International Conference on Smart Grid Communications (SmartGridComm2014), pp.782-78, 2014.

[10] M. Jung, T. Hofer, S. Döbelt, G. Kienesberger, F.Judex, and W. Kastner, "Access control for a Smart Grid SOA," in 2012 International Conference for Internet Technology and Secured Transactions, ICITST 2012, pp. 281-287, 2012.

[11] D. Chassin, and K. Kalsi, "Effects of Demand Response on Retail and Wholesale Power Markets", IEEE Power and Energy Society General Meeting 2012, pp.00-00, 2012.

[12] T. Fukazawa, A. Kiyota, and C. Ninagawa, "Aggregated Transfer Function for Smart Grid FastADR Feedback Control of Wide Area Distributed Building Facilities", IEEJ Trans. on Electrical and Electronic Engineering, Vol.10, pp.487-489, 2015.

[13] OpenADR Allience, "OpenADR 2.0 Profile Specification B Profile", Document No. 20120912-1, p14, 2013

[14] IEEE Standard 1888-2011, "IEEE standard for ubiquitous green community control network protocol," IEEE, New York, 2011.

[15] H. Ochiai, M. Ishiyama, T. Momose, N. Fujiwara, K. Ito, H. Inagaki, A. Nakagawa, and H. Esaki : "FIAP: Facility information access protocol for data-centric building automation systems," in Proc. IEEE INFOCOM 2011, Workshop on M2MCN-2011, pp. 229-234 , 2011.

[16] H. Ochiai : "Power Data Management on the Internet Space: Green ICT Project in Japan," IEEE Colombian Communication Conference COLCOM2012, pp.1-2, 2012.

[17] L. Rizzo, "Dummynet: A Simple Approach to the Evaluation of Network Protocols", ACM Computer Communication Review, Vol.27, No.1, pp.31-41, 1997.

[18] E. Gilbert, "Capacity of a burst-noise channel", Bell System Technical Journal, Vol.39, pp.12531265, 1960.

[19] E. Elliot, "Estimation of error rates for codes on burst-noise channels", Bell System Technical Journal, Vol.42, pp.1977-1997, 1963. 